\title{
Meta
}

Journal des traducteurs

Translators' Journal

\section{LADMIRAL, Jean-René et LIPIANSKY, Edmond Marc (2015) : La Communication interculturelle. Paris : Les Belles Lettres, 330 p.}

\section{Milouda Medjahed}

Volume 63, numéro 1, avril 2018

URI : https://id.erudit.org/iderudit/1050531ar

DOI : https://doi.org/10.7202/1050531ar

Aller au sommaire du numéro

Éditeur(s)

Les Presses de l’Université de Montréal

ISSN

0026-0452 (imprimé)

1492-1421 (numérique)

Découvrir la revue

Citer ce compte rendu

Medjahed, M. (2018). Compte rendu de [LADMIRAL, Jean-René et LIPIANSKY,

Edmond Marc (2015) : La Communication interculturelle. Paris : Les Belles

Lettres, 330 p.]. Meta, 63(1), 269-272. https://doi.org/10.7202/1050531ar

Ce document est protégé par la loi sur le droit d'auteur. L'utilisation des services d'Érudit (y compris la reproduction) est assujettie à sa politique d'utilisation que vous pouvez consulter en ligne.

https://apropos.erudit.org/fr/usagers/politique-dutilisation/
Cet article est diffusé et préservé par Érudit.

Érudit est un consortium interuniversitaire sans but lucratif composé de l’Université de Montréal, l'Université Laval et l'Université du Québec à Montréal. Il a pour mission la promotion et la valorisation de la recherche. https://www.erudit.org/fr/ 
cultural conditions that spawn translations. Besides interlingual translation, intralingual translation and intersemiotic translation are also included in post-translation studies. In addition, it can cover all the new forms and genres brought about by new media. Post-translation studies can include intersemiotic transfers such as cinematic, musical or other media versions and better study them.

Nevertheless, there are difficulties in carrying out post-translation studies. As Gentzler points out in his conclusion: "post-translation studies is not bound by fixed objects - source and target texts - but is more fluid and includes looking at the pre-textual components, the multilingual aspects and multicultural ideas that comprise an original" (p. 230). This process demands meticulous study and analysis, as well as a sharp mind. Moreover, post-translation repercussions are not easy to investigate: "The repercussions can be seen everywhere - in print, art, signage, fashion, food, and media - and in every discipline - literature, politics, architecture, anthropology, philosophy, and religion" (p. 231). So, comprehensive knowledge is needed to carry out such a study. Although Gentzler offers four case studies as guidance, there is not a fixed framework to follow in carrying out post-translation studies.

Despite these difficulties, post-translation studies will be a new trend. In order to make his argument forceful, Genztler has made great efforts in selecting and organizing his material. First, Genztler takes great trouble to select his four case studies, which cover a wide variety of cultural zones, namely England, Germany, France, Brazil, and China. They are also arranged chronologically: "beginning with ElizabethanAge British, followed by German romanticism, Belle Epoque French, and twentieth-century Chinese modernization" (p. 16), which makes each case study quite representative. Then, this book is quite impressive in its archeological work. There are 20 tables listing the translations, rewritings and adaptations of each work under study. These lists offer a very convincing argument for translation's place at the center of cultural circulation. It is also worth mentioning that this book is reader friendly. When discussing theatrical productions and films, pictures are provided to give the reader a direct visual perception.

Overall, Gentzler's Translation and Rewriting in the Age of Post-Translation Studies is a remarkable book. All his efforts will hopefully push translation studies forward and into a vast territory and into a new age. Researchers in translation studies and beyond will all benefit from this enlightening and challenging book.

JiNHUi LIU AND JUN WEN Beihang University, Beijing, China

\section{REFERENCES}

DAmrosch, David (2003): What is World Literature? Princeton: Princeton University Press.

NergaArd, Siri and Arduini, Stephano (2011): Translation: A New Paradigm. Translation. Inaugural Issue:8-17.

LADMIRAL, Jean-René et LiPIANSKy, Edmond Marc (2015): La Communication interculturelle. Paris: Les Belles Lettres, 330 p.

À la suite de l'épuisement de la première (1989), deuxième (1991) et troisième (1995) édition du présent ouvrage, une quatrième édition est parue en octobre 2015. Du côté du fond, cette édition prône l'osmose entre les études interculturelles et la traductologie, deux disciplines considérées à la fois connexes et distinctes dans les éditions antérieures. Quant à la forme, cette nouvelle édition a porté sur l'élimination des coquilles et l'amélioration de plusieurs formulations inadéquates tout en conservant la pagination originale.

Le seul compte rendu du livre réalisé jusqu'alors est celui de Judith Woodsworth (1990), dans $T T R$, à propos de la toute première édition de 1989, une bonne raison de se livrer de nouveau à l'exercice en se basant sur la dernière édition.

L'ouvrage est composé de trois parties et de onze chapitres. L'introduction porte sur la communication interculturelle comme enjeu et défi mondial, avec une attention particulière aux sociétés de l'Europe occidentale. Les auteurs définissent la notion de culture comme étant «les modes de vie d'un groupe social: ses façons de sentir, d'agir ou de penser; son rapport à la nature, à l'homme, à la technique et à la création artistique» (p. 8). Quant au terme «interculturel», il est défini comme "un champ interactif, où l'on s'interroge sur les relations qui s'instaurent entre groupes culturellement identifiés» (p. 10). L'interculturel ici traité s'intéresse particulièrement à l'exemple paradigmatique des relations entre Français et Allemands menées dans le cadre des "programmes de formation-recherche» commandités par l'OFAJ (Office franco-allemand pour la jeunesse). Pour ce faire, les auteurs font appel à une approche psychosociologique qui s'appuie sur la dynamique des groupes binationaux et plurinationaux et concilie pratique et empirique. Les résultats de recherche ainsi analysés posent les bases d'une analyse sémiotique et sociohistorique intitulée l'«imagologie interculturelle» (p. 15).

Dans le premier chapitre, "Pour une dynamique des groupes bilingues ", Ladmiral traite des aspects psychosociologiques du bilinguisme qu'il qualifie d' «inédits». Aidé par une équipe d'animation et de recherche bilingue et monolingue, 
il a étudié un groupe de 30 jeunes comprenant des unilingues francophones, des unilingues germanophones et des bilingues, vivant ensemble dans le cadre d'un stage résidentiel de huit jours. Des problèmes psychologiques émergent au cours du processus d'interprétation, appelé également «traduction orale». En effet, l'analyse des motivations à interpréter (traduire selon Ladmiral) révèle que cette "médiation linguistique» est devenue un enjeu de pouvoir linguistique, voire une source de conflit (on en vient, par exemple, à privilégier l'allemand au détriment du français). On observe aussi l'interprète qui fait appel à son bagage culturel pour effectuer une traduction sélective; la traduction joue alors le rôle d'un filtre de la communication interculturelle et devient elle-même la dynamique du groupe.

L'auteur rappelle que «la communication est un cas particulier du malentendu» (p. 74); elle se trouve d'une part, devant un obstacle d'ordre parolique ou verbal (plurilinguisme), et d'autre part, devant une autre difficulté d'ordre culturel (ou socioculturel) se situant dans le paraverbal.

Le deuxième chapitre, "Le corps entre deux langues», traite de la dimension corporelle vécue de la communication interculturelle. Ladmiral affirme que la voix humaine n'est que l'intersection, l'union paradoxale entre le corps, qui s'y spiritualise, et l'esprit, qui s'y incarne. Il se perd ensuite dans une discussion savante et métaphysique sur la voix humaine, cette "entité à deux faces", illustrée par de nombreuses références à saint Augustin, Kant, Hegel, Shelling, Barthes et Goethe.

Dans le troisième chapitre: «De la langue à la culture», Lipiansky survole d'abord les hypothèses sur la relation entre la langue et la culture (LéviStrauss, Sapir Whorf, entre autres) et les travaux sur la relation entre le langage, la réalité sociale préexistante et la culture (Kant, Ernst Cassirer, Lacan, entre autres). Ensuite, l'auteur affirme que le langage, qui n'est jamais neutre, est porteur de valeurs sur lesquelles une société bâtit sa culture et son identité nationale. Le langage devient un des enjeux d'une lutte de classes sociales utilisé pour faire reconnaître et imposer ses valeurs et sa culture. Pour illustrer ses propos, Lipiansky utilise l'exemple du terme civilisation et de sa traduction allemande Kultur, qui, replacés dans leur contexte historique, reflètent des représentations sociales très différentes et des conceptions antagonistes des valeurs et de l'identité nationales.

Dans le quatrième chapitre: "Identité et altérité ", Lipiansky aborde, à la lumière d'une approche génétique, la problématique de la similitude et de la différence dans les rapports interculturels. Il part de l'idée hégélienne de l'impossibilité de saisir la conscience de soi autrement qu'à travers la reconnaissance de celle de l'Autre pour arriver au rôle ambivalent de l'autrui dans la formulation de l'identité de soi selon la démarche expérimentale de Zazzo (1 ${ }^{\text {re }}$ édition 1980, $2^{\text {e }}$ édition 1986). Cette structuration des rapports entre identité et altérité (ici les différentes étapes fondamentales du développement psychologique du jeune enfant) est projetée sur des sociétés où les groupes entrent dans une lutte avec l'Autre, étranger, afin de se distinguer, de se reconnaître et de construire leur propre identité nationale, qui instaure la communication et l'échange.

Dans le cinquième chapitre: "L'ouverture à l'altérité culturelle», Lipiansly aborde le thème de la communication interculturelle et y étudie le phénomène de l'ethnocentrisme, constitutif de tout groupe d'individus, tout en soulignant l'importance d'un travail de décentration pour une ouverture à l'altérité.

Ensuite, «L'identité nationale dans la dynamique de la rencontre» explore le rôle de l'identité nationale subjective dans les communications interculturelles, et ceci, selon l'expérience de la dynamique des groupes plurinationaux. Lipiansky y expose brièvement diverses démarches méthodologiques et propose la dynamique des groupes comme mode d'investigation spécifique. Il avance alors une méthode qui soutient que «l'identité ethnique ou nationale s'actualise de façon privilégiée dans une situation où elle est confrontée à d'autres identités» (p. 186). Cette hypothèse est vérifiée au moyen de plusieurs expériences regroupant des jeunes venant de pays différents (majoritairement des étudiants) intéressés par les contacts interculturels. L'auteur analyse, pendant la rencontre, les réactions verbales et comportementales en relation avec l'identité nationale subjective de chaque sous-groupe national, et découvre l'intérêt d'une telle expérience pour l'évolution personnelle des individus.

Comme souligné par Lipiansky, toute rencontre interculturelle est caractérisée par la médiatisation des «représentations sociales» que les peuples se font les uns des autres. Découlant de l'imaginaire social, ces représentations sont le fruit de la tradition, mais aussi de la dynamique des rapports intergroupes. Le septième chapitre: «Représentations sociales et contacts interculturels» présente les bases d'une «imagologie interculturelle», une étude sémiotique et sociohistorique de ces représentations identitaires, basée sur la psychologie sociale. En effet, après avoir évoqué un historique des recherches expérimentales antérieures (les travaux de Sherif, Brunet et Perlmutter, Doise et Deschamps), l'auteur tente de dégager des «lois générales» propres à une situation historique. Des situations où la représentation et l'image de l'identité nationale d'une nation sont projetées sur celles des autres afin de saisir sa propre image. 
Cette analyse est illustrée par des exemples concrets tirés des rapports franco-allemands dans le chapitre suivant: "L'imagerie de l'identité, le couple France-Allemagne». Ces exemples sont limités à un ensemble de littérature d'essais européens consacrés aux caractères nationaux et datant de la première moitié du $\mathrm{xx}^{e}$ siècle. Il s'agit ici des essais qui représentent l'identité nationale française dans ses relations à l'identité allemande. L’objectif est «d'en montrer les présupposés, l'organisation et les processus internes, d'en saisir les modalités sociales, historiques et idéologiques de production, d'en dévoiler les fonctions dans la dynamique des relations sociales et internationales» (p. 212).

Dans le neuvième chapitre: «Éléments d'imagologie franco-allemande», Ladmiral propose une interprétation ethnopsychologique des images et des stéréotypes allemands, négatifs ou cultivés, au sein de la culture française. Il expose ensuite son analyse illustrée par de nombreux exemples des figures que prend la France dans l'histoire allemande (et inversement), notamment après le drame de 1870-1871. Il s'agit, en effet, d'une représentation qui fait objet d'une sexualisation des rapports franco-allemands à partir de 1870 1871, une imagerie constamment réactualisée et diagnostiquée par Ladmiral par "un complexe de Bismarck» de la conscience nationale française. Comme guérison, il ne préconise pas de refouler le passé, mais plutôt d'assumer les stéréotypes et de les approfondir.

Les deux derniers chapitres: «Dynamique d'un groupe en vacance(s) d'identité nationale» et «Le tabou du national» constituent un exemple concret de la difficulté d'aborder la problématique du national. En est donnée comme exemple l'interprétation du vécu d'un stage franco-allemand qui s'est déroulé du 6 au 16 juillet 1982 à OsterholzSharmbeck sur la problématique du national et de l'identité. Dans le but de briser le tabou de décentralisation nationale, l'équipe d'animation a proposé d'organiser le stage en trois étapes: une combinaison de "réflexion-discussion en sousgroupes, sa collectivisation critique en séance plénière [...] et un espace d'échanges informels en groupes de rencontres affinitaires» (p. 282). Toutefois, fuyant les difficultés d'une relation purement parolique, cette modalité de communication a été refusée par les stagiaires au profit de diverses pratiques qui privilégient l'«échéance du nonverbal». Ladmiral avance donc plusieurs interprétations psychosociologiques de cette méfiance envers le langage dont la principale explication est le refus de "parler (de) l'identité nationale», un refoulement du national qui conduit l'auteur à faire l'hypothèse d'une «occultation groupale de l'identité nationale».
Dans le dernier chapitre, Ladmiral souligne une "dénégation» du national lorsqu'il y a un désir, une pensée ou un sentiment d'un national refoulé par l'individu pour se défendre, pour afficher sa volonté d'ouverture à l'Autre et son désir de réconciliation ou encore pour «se fuir et se découvrir autre» (notamment du côté français). Il conclut son chapitre par des réponses aux éventuelles objections qu'on pourrait lui adresser tout en soulignant l'apport scientifique de son étude de cas aux problèmes de la traduction et à la dynamique des groupes bilingues.

Dans la conclusion, les auteurs soulignent l'importance d'une pédagogie de l'interculturel pour la pratique de la recherche et reprennent brièvement les plus importants apports théoriques de cette expérience concrète de la communication interculturelle.

Publié dans la collection «Traductologiques», l'ouvrage s'inscrit dans un cadre psychosociologique visant une pédagogie et la théorisation de la communication interculturelle. Le premier chapitre pourrait décevoir tous ceux qui espéraient trouver des réponses à certaines problématiques que pose l'activité traduisante. La principale cause de cette déception est le fait que, pour Ladmiral, traduction et interprétation se confondent, tout comme parole source/parole cible (p. 48) et texte source/texte cible (p.49), parole et texte d'un message (p. 53), dire et écrire (p. 53), traduire et interpréter (p. 53), etc. Quant aux chapitres rédigés par Lipiansky, ils ne touchent pas le domaine de la traduction, mais plutôt celui de la psychologie sociale.

Par ailleurs, l'ouvrage recèle un problème de cohérence du fait de l'approche différente des deux auteurs. On peut constater ainsi de nombreuses répétitions, telles que l'idée de se découvrir à travers l'Autre selon la vision hégélienne développée par Ladmiral dans le deuxième chapitre et reprise par Lipiansky dans le quatrième chapitre (voir p. 119-121).

La question des références bibliographiques mérite également quelques remarques. D’une part, on y trouve parfois un mélange de protocoles: les auteurs citent leurs références dans le corps du texte ou en bas de page (p. 201, 240-241, 266). D'autre part, certaines références sont absentes de la bibliographie, notamment celles de Zazzo (1986), de Cassirer (1969), de Curtius (1941), de Brunet et de Perlmutter (1979), ou encore de Gaultier (1936), qui sont bien insérées en bas de page (sauf pour Zazzo et Sapir et Whorf), mais ne figurent pas dans la bibliographie de l'ouvrage.

Il reste toutefois à souligner que Ladmiral a le mérite de soulever des problèmes importants, voire fondamentaux, que pose l'interprétation, tels que l'allongement, la question des non-dits, le sujet 
traduisant, la décision d'interpréter dans le cas de sensibilité "ethnolinguistique», ou encore l'(in) traduisibilité de l'humour.

Milouda Medjahed

Université de Montréal, Montréal, Canada

\section{RÉFÉRENCES}

Bruner, Jerome et Perlmutter, Howard (1979): Compatriotes et étrangers: une étude de formation des impressions dans trois pays. In: Willem Doise (dir.). Expérience entre groupes. (Traduit par Jacqueline Deschamps) Paris: École des hautes études en sciences sociales/Mouton Éditeur, 227-242.

CAssirer, Ernst (1969): Le langage et la construction du monde des objets. In: Jean-Claude Pariente (dir.). Essais sur le langage. Paris: Éditions de Minuit, 37-68.

Curtius, Ernst-Robert (1941): Essais sur la France. Paris: Grasset.

Gaultier, Paul (1936): L'âme française. Paris: Flammarion.

SAPIR, Edward (1921): An Introduction to the Study of Speech. New York: Harcourt, Brace and Company.

Whorf, Benjamin Lee (1956): Language, Thought and Reality. Selected Writings of Benjamin Lee Whorf. (Édité par John Bissel Carroll, Stephen Curtis Levinson et Penny Lee) Cambridge: The MIT Press.

Woodsworth, Judith (1990): Compte rendu de La Communication interculturelle par Jean-René Ladmiral et Edmond Marc Lipiansky [1989, Paris: Armand Collin]. TTR. 3(1):133-136.

ZAzzo, René (1986): Les dialectiques originelles de l'identité. In: Pierre TAP (dir.). Identité individuelle et personnalisation. $2^{\mathrm{e}}$ éd. Toulouse: Privat.

Mikkelson, Holly et Jourdenais, Renée (dir.) (2015): The Routledge Handbook of Interpreting. Londres/New York: Routledge, 456 p.

Le Routlege Handbook of Interpreting aborde l'interprétation professionnelle sous les quatre angles suivants: 1) perspective historique; 2) modes et situations d'interprétation; 3) cadres spatio-temporels; et 4) problématiques et débats. Constitué de 12 pages préliminaires et de 456 pages de contenu, il a été rédigé par 31 contributeurs. Le volume explore les multiples facettes de la profession d'interprète, des points de vue théorique et pratique. Les chapitres se succèdent selon une même structure, soit un aperçu historique, un état actuel et une perspective d'avenir. Le volume procède selon un axe chronologique logique, de sorte que le lecteur se repère facilement dans un schéma évolutif graduel. La qualité de l'inter- prétation dans les divers cadres spatio-temporels qu'englobe la profession constitue la pierre angulaire de l'ouvrage.

La première partie passe au crible le développement de la pratique interprétative depuis l'Égypte antique jusqu'à la Deuxième Guerre (Jesús Baigorri-Jalón); la participation, sur le plan professionnel, des organisations internationales et académiques dans la détermination des cadres de la profession (Julie Boéri); l'influence de la géopolitique internationale sur le développement de cette dernière (Sofia García-Beyaert); ainsi que le tournant pris par l'interprétation en matière de recherche axée sur les avantages et inconvénients de la technologie mise au service du domaine (Franz Pöchhacker).

Les modes d'interprétation se réfèrent spécifiquement aux pratiques sur le terrain et les compétences nécessaires à la bonne maîtrise de chaque mode, soit: l'interprétation simultanée (Kilian Seeber et Debra Russell) et l'interprétation consécutive (Kayoto Takeda). La langue des signes, l'interprétation à vue (sight interpreting) ainsi que la transcription du discours parlé (spoken language interpreting, SLI) sont discutées respectivement par Karen Bontempo et Jemina Napier, Wallace Chen et Carmen Valero-Garcés.

Les neuf chapitres de la troisième partie traitent des cadres spatio-temporels régissant l'interprétation, par exemple: le développement de l'interprétation de conférence (Ebru Diriker), thème faisant écho à l'article de Julie Boéri dans la première partie; l'interprétation judiciaire et les questions éthiques s'y rapportant (Jieun Lee); la problématique des questions morales et éthiques dans les procédures de demande d'asile (Sonja Pöllabauer); l'interprétation communautaire (Marjory A. Bancroft) qui exige la médiation linguistique et culturelle de l'interprète; l'interprétation se rapportant aux soins de santé et de santé mentale (Cynthia E. Roat et Hanneke Bot, respectivement) ainsi que les questions éthiques qu'elle soulève; et l'interprétation dans les domaines de l'éducation et des médias (Melissa B. Smith et Pedro Castillo).

Parmi ces nombreuses contributions, nous en retiendrons deux, celles de Barbara Moser-Mercer, Interpreting in conflict zones (p. 302-316), et celle de Uldis Ozolins, Ethics and the Role of the Inter$\operatorname{preter}($ p. 319-336), pour leur manière intéressante d'aborder l'éthique. Le premier article décrit le rôle critique des interprètes en zone de conflit, ainsi que les défis auxquels ils font face, et le problème qui survient lorsque les organisations qui font appel à ces interprètes dits sur le terrain (p. 302) comprennent mal le métier de l'interprétation et embauchent des novices. L'article souligne surtout la complexité légale de la situation des interprètes en zone de conflit. La question qui revient souvent 